\title{
Inhibition of prostate cancer growth by immunization with a GM-CSF-modified mouse prostate cancer RM-1 cell vaccine in a novel murine model
}

\author{
HONGMEI XIA, XIAOJING LUO and WEIHUA YIN \\ Department of Oncology, The People's Hospital of Yichun Affiliated to Clinical Medicine School of \\ Yichun University in Jiangxi Province, Yichun, Jiangxi 336000, P.R. China
}

Received May 25, 2016; Accepted September 1, 2017

DOI: $10.3892 / \mathrm{ol} .2017 .7332$

\begin{abstract}
Advanced prostate cancer is difficult to treat owing to a lack of effective approaches for disrupting immune tolerance. C57BL/6 male and female mice implanted with viable RM-1 cells represent a novel murine model of advanced prostate cancer for studying antitumor effects following immunization with a granulocyte-macrophage colony-stimulating factor (GM-CSF)-modified RM-1 cell vaccine, which has been described previously. In vitro cytotoxic activity and cytokine secretion experiments were conducted to investigate the antitumor response. The cytotoxicity profile of splenocytes from female mice immunized against RM-1 cells primarily involved cytotoxic T lymphocyte (CTL) lysis and, to a lesser extent, natural killer (NK) cell lysis. NK cell lysis was also observed in males, which exhibited no evidence of CTL lysis. The secretion of interferon- $\gamma$ in the GM-CSF-modified cell vaccine group was significantly increased compared with the other groups. The level of interleukin-4 was low. To investigate the antitumor immune response further, cluster of differentiation 4 (CD4) T cells and CD8 T cells were analyzed in the spleens and tumors of female mice receiving the GM-CSF-modified RM-1 cell vaccine. Unlike female mice, males exhibited the highest proportion of NK cells in the spleen. NK cells were not detected in the tumor tissue in any of the groups. The difference between the sexes may explain the specificity of the immune response, as females are intolerant to prostate antigens whereas males are. This model is clinically relevant as it translates to human immunology and offers an
\end{abstract}

Correspondence to: Dr Weihua Yin, Department of Oncology, The People's Hospital of Yichun Affiliated to Clinical Medicine School of Yichun University in Jiangxi Province, 88 Zhongshan West Road, Yichun, Jiangxi 336000, P.R. China

E-mail: ywh1939@163.com

Key words: granulocyte-macrophage colony-stimulating factormodified RM-1 cell vaccine, prostate cancer, a novel murine model mode, immune response effective and convenient method for studying immunotherapy for prostate cancer.

\section{Introduction}

Prostate cancer is a common disease in the Western world, with one in five men suffering from prostate cancer in the United States (1). Early-stage prostate cancer is usually treated via radical prostatectomy and radiation therapy; however, there is no effective therapy once metastatic prostate cancer is diagnosed (2). The US Food and Drug Administration suggest that docetaxel should be used to treat advanced prostate cancer, and under this treatment median survival can be improved by 2-4 months $(3,4)$. However, docetaxel causes numerous side effects, meaning a novel effective and nontoxic treatment is required.

Whole-tumor cell vaccines have been extensively studied in melanoma (5) and lung cancer (6). Various methods have been developed for antigen preparation, including the use of $\gamma$-irradiated tumor cells, formalin-fixed cells $(7,8)$, glutaraldehyde-fixed cells (9) and frozen/thawed cells (10). As cellular proteins are better preserved in ethanol-fixed tissue $(8,11)$, ethanol-fixed RM-1 cells were selected as a source of tumor antigens for immunotherapy.

The RM-1 prostate cancer cell line is derived from urogenital sinus cells from tumor protein 53-knockout C57BL/6 mice, and was transformed with Ras proto-oncogene GTPase (Ras) and MYC proto-oncogene BHLH transcription factor (Myc) (12). It is aggressive, non-immunogenic and expresses low levels of major histocompatibility complex I (MHCI) (12). As RM-1 cells express low levels of MHCI, it is difficult for $\mathrm{T}$ cells to mediate an antitumor response in male C57BL/6 mice. Griffith et al (13) demonstrated that male mice immunized with $\gamma$-irradiated RM-1 cell vaccines failed to clear viable RM-1 cells (13), likely owing to prostate antigen tolerance following whole-cell vaccination. If this were the case, females would be well-protected following vaccination, as females are intolerant to prostate antigen (14). The difference in the immune response can presumably be attributed to antigen tolerance based on sex differences. If the sex difference was demonstrated to mediate immunity in these mice, they may be used to study the effects of different immunotherapies, 
including ethanol-fixed cell vaccines combined with novel adjuvants or cytokines. It is likely that protection can be improved in males following vaccination. To investigate the antitumor response, a novel platform was established based on the unique property of streptavidin (SA) to bind rapidly and irreversibly to biotin-linked molecules, and the ability of biotin to be readily incorporated into the proteins on the cell surface. This allows for the rapid $(<2 \mathrm{~h})$, efficient and durable display of SA-tagged bioactive cytokines on the surface of biotinylated tumor cells. A granulocyte-macrophage colony-stimulating factor (GM-CSF)-surface-modified RM-1 cell vaccine was generated based on this technology, which has previously been shown to be effective in inducing antitumor immunity $(8,15)$. In the present study, C57BL/6 female and male mice were injected with viable RM-1 cells as a novel tumor model to study the mechanisms of antitumor immunity. This model makes it possible to identify an effective and convenient method for studying immunotherapies for prostate cancer.

\section{Materials and methods}

Animals and cells. C57BL/6 mice (n, 60; 30 female and 30 male) were purchased from the Animal Experiment Center of the Southern Medical University (Guangzhou, China). The mice were housed under specific pathogen-free conditions. Cages, bedding, food and water were autoclaved and changed regularly (food and water was added every morning and evening). The mice were maintained in a 12:12 h light:dark cycle. All the mice used for the study were at 6-8 weeks of age. All animal studies were approved by the Experimental Animal Ethics Committee of the People's Hospital of Yichun (Yichun, China) and were performed in accordance with the Regulations for the Administration of Experimental Animals in China published in 1988.

RM-1 prostate cancer cells were provided by the Southern Medical University, (Guangdong, China). The cells were cultured in Dulbecco's modified Eagle's medium (DMEM) supplemented with $10 \%$ fetal bovine serum (FBS; Gibco; Thermo Fisher Scientific, Inc., Waltham, MA, USA), $100 \mathrm{U} / \mathrm{ml}$ penicillin, and $100 \mu \mathrm{g} / \mathrm{ml}$ streptomycin. The cells were maintained at $37^{\circ} \mathrm{C}$ in a humidified atmosphere of $5 \% \mathrm{CO}_{2}$.

Preparation of the GM-CSF-surface-modified RM-1 cell vaccine. The GM-CSF-surface-modified RM-1 cell vaccine was prepared as previously described $(8,15)$. Briefly, RM-1 cancer cells were harvested using trypsin, washed twice with sterile PBS, incubated with $30 \%$ ethanol (v/v) at room temperature for $1 \mathrm{~h}$, washed twice with PBS, counted using $0.4 \%$ trypan blue and finally resuspended at $2 \times 10^{7}$ cells $/ \mathrm{ml}$ in PBS. Ethanol-fixed RM-1 cells $\left(2 \times 10^{7}\right.$ cells $\left./ \mathrm{ml}\right)$ were incubated with 10 mM-fresh EZ-Link Sulfo-NHS-Biotin (Pierce; Thermo Fisher Scientific, Inc.) at room temperature for 30 min with $4 \mu \mathrm{g}$ of $6 \mathrm{xHis-L-SA-GM-CSF}$ fusion protein, which was prepared in our laboratory $(8,15)$. Subsequent to three washes with PBS, the biological activity of the modified SA-GM-CSF on the cell surface was assayed by bone marrow cell proliferation as previously described $(8,15)$.

Tumor model and immunization.C57BL/6 mice were implanted with viable RM-1 prostate cancer cells. C57BL/6 males were injected intradermally with $2 \times 10^{6}$ GM-CSF-modified cells (as the vaccine), ethanol-fixed cells or PBS in the right thigh at weekly intervals for three consecutive weeks. At 1 week after the final vaccination, the mice were challenged with a subcutaneous injection of $1 \times 10^{5}$ viable RM-1 cells suspended in $100 \mu \mathrm{l}$ of PBS in the left flank. Tumor growth was measured 2-3 times/week with a Vernier caliper. The animals were sacrificed when the tumors either reached a diameter of $20 \mathrm{~mm}$ or exhibited ulceration. Male and female mice were treated equally. All experiments were repeated three times using groups of 10 mice.

Cytotoxic activity assay. Splenocytes were isolated from the experimental mice following the second vaccination and 2 weeks after the last immunization. Red blood cells were lysed with ammonium-chloride-potassium (ACK) lysis buffer (0.15 $\mathrm{M} \mathrm{NH}_{4} \mathrm{CL}, 1 \mathrm{mM} \mathrm{KHCO}_{3}$, and $0.1 \mathrm{mM} \mathrm{NaETDA}, \mathrm{pH}$ 7.2). The splenocytes were resuspended in DMEM containing $10 \%$ FBS, following which recombinant human interleukin-2 (IL-2; R\&D Systems China Co., Ltd., Shanghai, China) was added and RM-1 cells were subjected to $25 \mu \mathrm{g} / \mathrm{ml}$ mitomycin C (Boster Biological Technology, Pleasanton, CA, USA) incubation for 5 days at room temperature. Re-stimulated effector $\mathrm{T}$ cells were collected using a discontinuous Ficoll-Hypaque gradient by two centrifugations, each for $10 \mathrm{~min}$ at $289 \mathrm{x} \mathrm{g}$ at room temperature, and the concentration was adjusted to $1 \times 10^{6}$ cells $/ \mathrm{ml}$ in DMEM. Target RM-1 cells were seeded at $1 \times 10^{4}$ cells in $100 \mu \mathrm{l}$ of medium per well in 96 -well plates. The target RM-1 cells were subsequently added to effector T cells at various effector-to-target ratios and cultured for $4 \mathrm{~h}$ at $37^{\circ} \mathrm{C}$. The supernatant was collected to measure lactate dehydrogenase activity using the CytoTox $96^{\circledR}$ Non-Radioactive Cytotoxicity assay (Promega Corporation, Madison, WI, USA). The percentage of cytotoxicity was calculated as follows: 100x (experimental-effector spontaneous-target spontaneous)/(target maximum-target spontaneous).

Purifying cluster of differentiation $8(C D 8)^{+}$T cells. CD8 $\mathrm{a}^{+}$ T cells were isolated from murine splenocytes using the CD8 $\mathrm{a}^{+}$ T cell Isolation kit (Miltenyi Biotec GmbH, Bergisch Gladback, Germany). Splenocytes were resuspended at $1 \times 10^{7}$ cells $/ 40 \mu 1$ of buffer [PBS containing $0.05 \%$ bovine serum albumin (BSA); Sigma-Aldrich; Merck KGaA, Darmstadt, Germany and $2 \mathrm{mM}$ EDTA, $\mathrm{pH}$ 7.2]. A biotin-antibody cocktail (Miltenyi Biotec $\mathrm{GmbH}$ ) was added at $10 \mu \mathrm{l} / 1 \times 10^{7}$ total cells, followed by mixing and incubation for $10 \mathrm{~min}$ at $4^{\circ} \mathrm{C}$. Next, the cells were cultured with $30 \mu \mathrm{l}$ of buffer and $20 \mu \mathrm{l}$ of anti-biotin microbeads (Miltenyi Biotec $\mathrm{GmbH}$ ) added to $1 \times 10^{7}$ cells at $4^{\circ} \mathrm{C}$ for $15 \mathrm{~min}$, washed once with buffer, centrifuged at $300 \mathrm{x}$ g for $10 \mathrm{~min}$, and resuspended at $1 \times 10^{8}$ cells $/ 500 \mu \mathrm{l}$ of buffer. Finally, the cell suspension was pipetted onto an MS column (Miltenyi Biotec $\mathrm{GmbH}$ ), and $\mathrm{CD} 8 \mathrm{a}^{+} \mathrm{T}$ cells were passed through the column.

Interferon- $\gamma(I F N-\gamma)$ and IL-4 ELISAs. Splenocytes were isolated from mice prior to vaccination and 7 days after the last vaccination. The splenocytes were purified as aforementioned using a CD8 $\mathrm{a}^{+} \mathrm{T}$ cell isolation kit and subsequently co-cultured with $20 \mathrm{U} / \mathrm{ml}$ recombinant human IL-2 for $48 \mathrm{~h}$. The culture supernatants were collected, and the levels of 
A

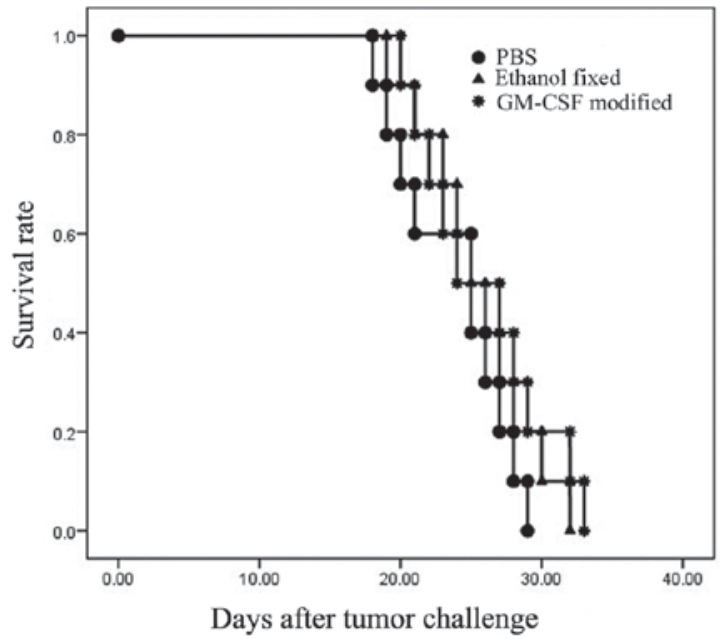

B

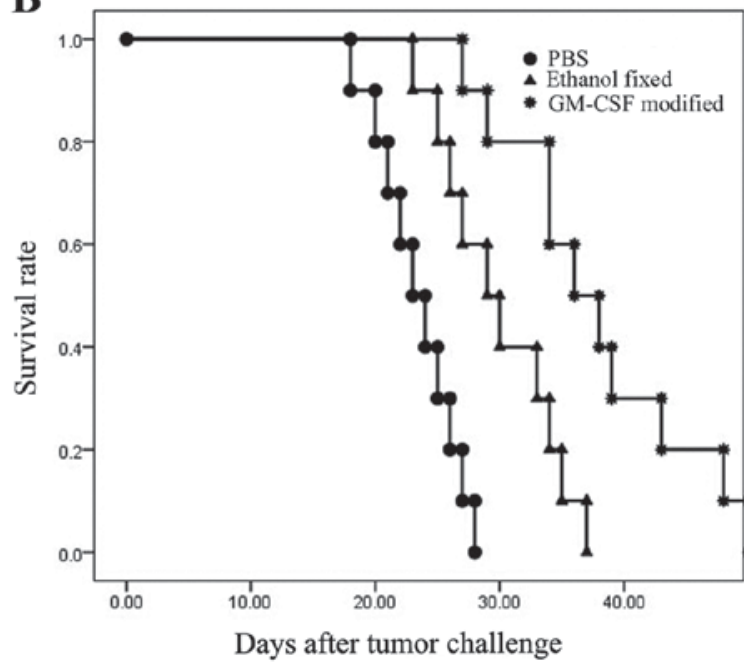

Figure 1. Survival analysis of the tumor model. C57BL/6 mice (10 mice per group) were vaccinated with $2 \times 10^{6}$ GM-CSF-modified cells or ethanol-fixed cells, or with PBS three times at weekly intervals. The mice were then subcutaneously injected with viable RM-1 cells (1x10 5 cells in $100 \mu 1$ PBS) 7 days after the final vaccination. (A) Experiments using males demonstrated that there was no significant difference between animals vaccinated with the GM-CSF-modified cells, ethanol-fixed cells and PBS groups $(\mathrm{P}=0.543)$. (B) Experiments using females demonstrated that two out of ten mice exhibited tumor-free survival at 45 days in the group vaccinated with GM-CSF-modified cells. The group receiving the vaccine exhibited significantly longer survival compared with the groups vaccinated with ethanol-fixed cells or PBS $(\mathrm{P}<0.05)$. Taken together, the data demonstrated that the survival curves were significantly different between female and male mice vaccinated with GM-CSF-modified RM-1 cells. P<0.05 for female mice vaccinated with GM-CSF-modified RM-1 cells vs. male mice vaccinated with GM-CSF-modified RM-1 cells.

IFN- $\gamma$ and interleukin IL-4 were measured via ELISA (R\&D Systems China Co., Ltd.).

Splenocyte analysis. Splenocytes were isolated from each experimental group on day 21 after tumor injection, then added to ACK lysis buffer to lyse red blood cells, washed twice with PBS with $1 \%$ BSA and incubated with fluorescein isothiocyanate (FITC)-labeled anti-mCD4, anti-mCD8 and anti-mCD161 antibodies (R\&D Systems China Co., Ltd.) for $1 \mathrm{~h}$ at room temperature. $\mathrm{CD} 4^{+} \mathrm{T}$ cells, $\mathrm{CD} 8^{+} \mathrm{T}$ cells, and $\mathrm{NK}$ cells were then analyzed via flow cytometry [BD Biosciences, Franklin Lakes, NJ, USA; FACS Vantage product with the Cell Quest software system (BD Cell Quest ${ }^{\mathrm{TM}}$ Pro version 6.0; BD Biosciences) was used for analysis].

Immunohistochemistry. Tumor samples from mice were snap-frozen in liquid nitrogen in Tissue Tec OCT compound (Boster Biological Technology). Frozen sections $(5-8 \mu \mathrm{m})$ were fixed in cold acetone at $4^{\circ} \mathrm{C}$ for $15 \mathrm{~min}$ and then washed with PBS and stained with anti-mCD4 (cat no. 553647; BD Pharmingen; BD Biosciences), anti-mCD8 (cat no. 553027; BD Pharmingen; BD Biosciences) and anti-mCD161 (cat. no. 566306; BD Pharmingen; BD Biosciences) overnight at $4^{\circ} \mathrm{C}$, according to the manufacturer's protocol for the HRP detection IHC kit (BD Biosciences). Dilution of the antibodies was 1:100 for anti-mCD4 and anti-mCD8 and 1:200 for anti-mCD161. The secondary antibody used was anti-rat IgG SABC kit (cat. no. BA1005; Boster Biological Technology, Pleasanton, CA, USA). After culture for $30 \mathrm{~min}$ at $37^{\circ} \mathrm{C}$, immunoreactivity products were visualized with a chromogenic agent 3,3'-diaminobenzidene, color development was performed for $<10 \mathrm{~min}$ until the desired color intensity was achieved at room temperature. Counterstaining was performed with hematoxylin for 1-2 min at room temperature. Positive staining cells were counted in a blind manner using an inverted microscope (magnification, x200; Leica Microsystems GmbH, Wetzlar, Germany). The results were recorded as the number of immunopositive cells per square millimeter.

Statistical analysis. Statistical analysis was performed using SPSS version 13.0 (SPSS, Inc., Chicago, IL, USA). All descriptive statistical data were presented as mean \pm standard deviation. The survival of mice was analyzed using Kaplan-Meier survival analysis and the log-rank test. For in vitro experiments, significant differences were determined using the Student's t-test and one-way analysis of variance, followed by Dunnett's post hoc test for multiple comparisons. $\mathrm{P}<0.05$ was considered to indicate a statistically significant difference.

\section{Results}

Survival of males and females. Male C57BL/6 mice were administered the GM-CSF-modified, ethanol-fixed RM-1 cell vaccine or the PBS control three times at weekly intervals. At 7 days after the final vaccination, the mice were challenged with $1 \times 10^{5}$ viable RM- 1 cells. In males, all mice developed tumors within 12 days and were sacrificed within 35 days. There were no significant differences between the GM-CSF-modified, ethanol-fixed and PBS (control) groups ( $\mathrm{P}=0.543$; Fig. 1). However, mice vaccinated with the GM-CSF-modified cells survived slightly longer compared with the mice from the other groups. In the females, two out of ten mice that were vaccinated with GM-CSF-modified cells exhibited tumor-free survival up to 45 days. All of the control mice formed tumors within 15 days. The survival curve was significantly different between females that were administered GM-CSF-modified cells, ethanol-fixed cells and PBS $(\mathrm{P}<0.001)$. Taken together, 

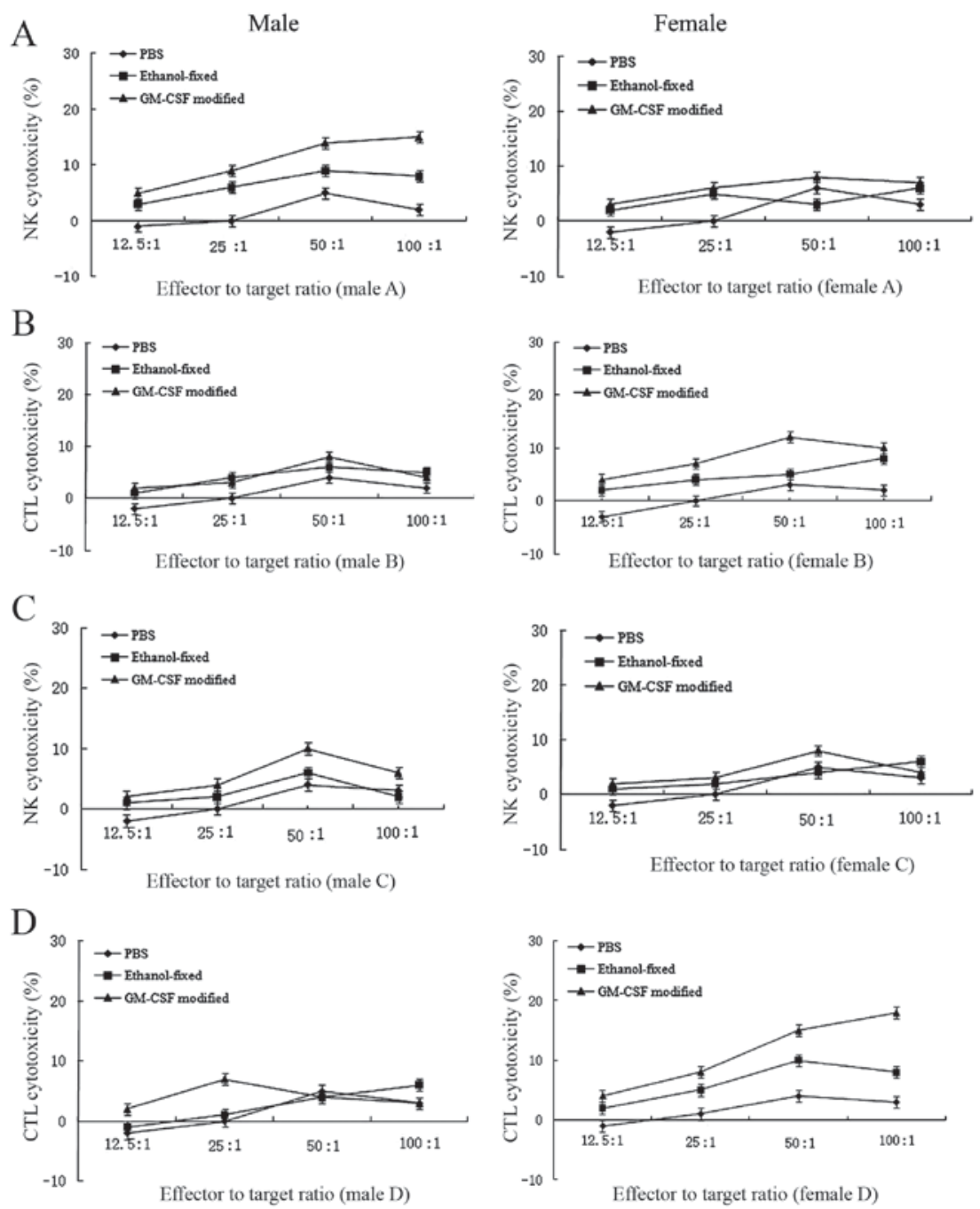

Figure 2. Cytotoxicity activity assay. Splenocytes were isolated from C57BL/6 mice following (A and B) the second vaccination and (C and D) 2 weeks after the third vaccination. In vitro cultures were performed as described in the Materials and methods section. Splenocytes were cultured with recombinant human interleukin-2 $(500 \mathrm{ng} / \mathrm{ml})$, and RM-1 cells were treated with $25 \mu \mathrm{g} / \mathrm{ml}$ mitomycin C for 5 days. The CytoTox 96R Non-Radioactive Cytotoxicity assay was used to measure lactate deydrogenase activity in the supernatant. As expected, cytotoxicity activity was undetectable in all control groups. In vaccinated males, low levels of NK cell cytotoxicity were detected in (A) and CTL lysis was undetectable in (D). In vaccinated females, the observed cytotoxic response was due to NK cell and CTL lysis. As shown in (B) and (D), the percent of cytotoxicity induced by CTLs was higher compared with that induced by NK cells. NK, natural killer; CTL, cytotoxic T lymphocyte; GM-CSF, granulocyte-macrophage colony-stimulating factor.

these results demonstrated that $\mathrm{C} 57 \mathrm{BL} / 6$ males were not protected following vaccination. By contrast, $20 \%$ of females were protected $(\mathrm{P}<0.05$; Fig. 1).

In vitro evaluation of the immune response. To analyze the antitumor immune response, the cytotoxicity of splenocytes against RM-1 cells from the experimental animals was examined using a nonradioactive cytotoxicity assay (Fig. 2). The cytotoxic response was collectively caused by NK cell and cytotoxic T lymphocyte (CTL) lysis. In the early stage (16), cytotoxicity was predominantly due to NK cell lysis. However, in the late stage, cytotoxicity was primarily mediated by CTL lysis. In the PBS group, CTL or NK cell activities were undetectable. In females, the antitumor response induced by the GM-CSF-modified cell vaccine was primarily mediated by CTL lysis and, to a lesser extent, by NK cell lysis. By contrast, males receiving the GM-CSF-modified RM-1 cell vaccine exhibited minor NK activity during the early stage following immunization. Additionally, CTL lysis was undetectable. The number of NK cells was reduced in the late stage, and these cells exhibited difficulty in mediating an antitumor response, which may explain the lack of protection afforded by the vaccine observed in males (Fig. 2).

$I L-4$ and $I F N-\gamma$. Splenocytes isolated with the CD8 $\mathrm{a}^{+} \mathrm{T}$ cell isolation kit were cultured with recombinant human IL-2 for $48 \mathrm{~h}$. The supernatants were harvested and examined via ELISA (Fig. 3). As expected, the levels of cytokines IL-4 and IFN- $\gamma$ in the supernatant were low to undetectable in the PBS group. The secretion of IFN- $\gamma$ was significantly increased following three cycles of immunization in the GM-CSF-modified cell vaccine group compared with the other groups $(\mathrm{P}<0.05)$. However, the secretion of IL-4 exhibited no significant differences during the course of vaccine therapy $(\mathrm{P}>0.05)$. In males, the levels of IFN- $\gamma$ secretion observed in mice receiving GM-CSF-modified cell vaccine, ethanol-fixed cells and PBS were $12.85 \pm 1.01,8.06 \pm 0.49$, and $4.76 \pm 0.23 \mathrm{pg} / \mathrm{ml}$, respectively. The supernatant levels of 
A

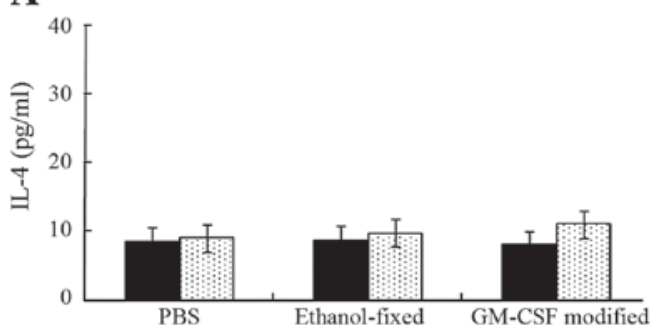

C

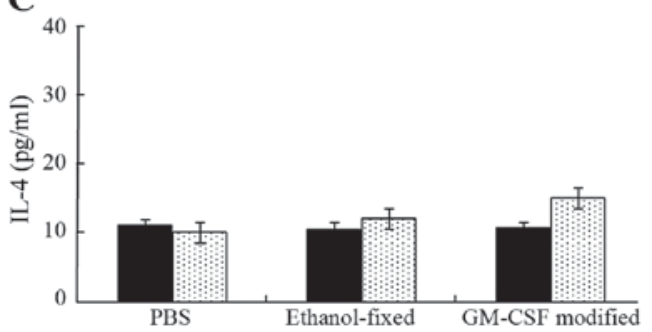

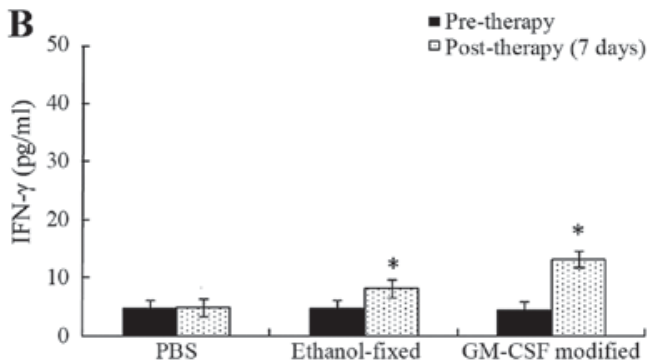

D

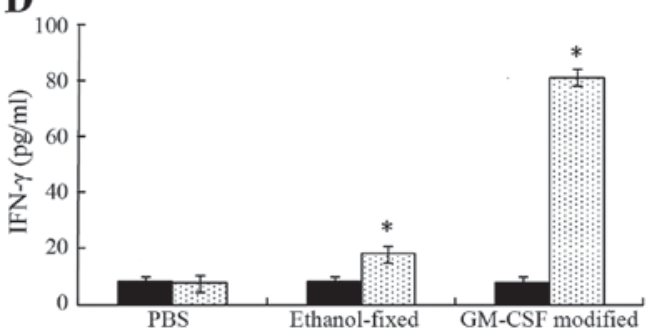

Figure 3. In vitro characterization of splenoctytes from experimental mice. Splenocytes were isolated from mice pre-vaccination and 7 days after the last vaccination. Splenocytes were purified with a CD8a ${ }^{+} \mathrm{T}$ cell isolation kit and then cultured with rhIL2 for $48 \mathrm{~h}$. The supernatants were collected and analyzed via ELISA. (A and B) Experiments conducted in male mice; (C and D) experiments conducted in female mice. The level of IL-4 in the supernatants was extremely low and exhibited no significant difference during the course of the vaccine therapy $(\mathrm{P}>0.05)$. However, the level of IFN- $\gamma$ in the supernatants was significantly increased following vaccine treatment compared with pre-vaccination. ${ }^{*} \mathrm{P}<0.05$ for pre-vaccination vs. post-vaccination. In graph $\mathrm{B}$ secretion of IFN- $\gamma$ measured in mice that received ethanol-fixed cells or PBS $(8.06 \pm 0.49$ and $4.76 \pm 0.23 \mathrm{pg} / \mathrm{ml}$, respectively). The secretion of IFN- $\gamma$ in the vaccine groups was higher than that in the PBS group $(\mathrm{P}=0.01)$. (D) The secretion of IFN- $\gamma$ in the ethanol-fixed cells and PBS groups was $17.47 \pm 1.51$ and $7.47 \pm 1.12$ pg/ml, respectively. IFN- $\gamma$ levels in the supernatants from vaccinated groups were significantly higher compared with those in the $\mathrm{PBS}$ group $(\mathrm{P}=0.001)$. As shown in (B and D), the secretion of IFN- $\gamma$ in males was lower compared with that in females. Standard deviation shown. CD8, cluster of differentiation 8; IL-4, interleukin-4; IFN- $\gamma$, interferon- $\gamma$.

IFN- $\gamma$ in the vaccine-treated group were significantly higher compared with the ethanol-fixed or PBS groups $(\mathrm{P}<0.05)$. In females, the supernatant levels of IFN $-\gamma$ in mice vaccinated with the GM-CSF-modified cell vaccine, ethanol-fixed cells and PBS was $80.34 \pm 3.01,17.47 \pm 1.51$, and $7.47 \pm 1.12 \mathrm{pg} / \mathrm{ml}$, respectively. The secretion of IFN $-\gamma$ was reduced following vaccination in males compared with females. These results indicated that the females presented a Th1 cytokine profile (Fig. 3).

Assessing CD4 T cells, CD8 T cells, and NK cells following vaccination. Splenocytes were isolated from the experimental mice on day 21 following the final vaccination and incubated with FITC-labeled anti-mCD4, anti-mCD8 and anti-mCD161 antibodies for $1 \mathrm{~h}$. The proportions of $\mathrm{CD} 4^{+} \mathrm{T}$ cells, $\mathrm{CD} 8^{+} \mathrm{T}$ cells and NK cells in the spleen were assessed via flow cytometry (Fig. 4A). The proportions of $\mathrm{CD}^{+}$and $\mathrm{CD}^{+} \mathrm{T}$ cells in the GM-CSF membrane-modified cell vaccine group were significantly higher compared with the other groups, with the proportions of $\mathrm{CD}^{+} \mathrm{T}$ cells and $\mathrm{CD} 8^{+} \mathrm{T}$ cells being higher in female spleens compared with in male spleens. However, the number of NK cells in females was lower compared with that in male mice, with NK cells not detectable in tumor tissue (Fig. 4A). The infiltration of $\mathrm{CD}^{+} \mathrm{T}$ and $\mathrm{CD} 8^{+} \mathrm{T}$ lymphocytes in the tumor tissue was examined via immunohistochemistry. Large numbers of $\mathrm{CD} 4^{+} \mathrm{T}$ cells and $\mathrm{CD}^{+} \mathrm{T}$ cells were identified in females administered with the GM-CSF membrane-modified cell vaccine (Fig. 4B). The data indicated that the GM-CSF membrane-modified cell vaccine may enhance antitumor immunity by increasing the numbers of T lymphocytes and NK cells, and that the observed difference in immunity was largely due to sex differences.

\section{Discussion}

Current methods of treatment for advanced prostate cancer have only limited success, at least in part due to an incomplete understanding of the immunobiology of human prostate cancer (17). A model that accurately mimics the human situation would therefore be useful for understanding antitumor immune responses in humans, and may offer a predictive model for therapeutic efficacy. For the past 10 years, the Transgenic Adenocarcinoma of the Mouse Prostate model has been used; however, this model is not appropriate to study how immunogenic viral oncogenes induce tumorigenicity (18-20). There is currently no effective model for advanced prostate cancer that can be used to fully explain antitumor immunobiology in humans. Thus, a novel model of prostate cancer that may be used to investigate how the tolerance of tumors can be disrupted to generate a sustained and potent immune response is required. Mice injected with viable RM-1 cells have been used as an advanced prostate cancer model as RM-1 cells are aggressive and non-immunogenic, expressing very low levels of MHCI $(12,20)$. In the present study, male and female mice injected with viable RM-1 cells were used as a novel animal model to assess immune responses to vaccination. The results indicated that the tested vaccine induced a stronger antitumor immune response in females than in males, and this result may be associated with immune tolerance. Only males have prostates, and prostate antigens are therefore recognized as 'self' antigens by the male immune system. In fact, RM-1 cells express the Myc and Ras oncogenes (12), which do not induce tolerance in males. Tolerance to an antigen is determined by the antigen's expression level (21). If the level of an antigen expressed by tumor cells is too low or too high, 


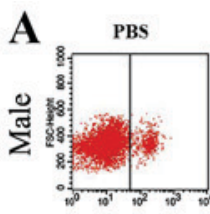

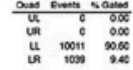

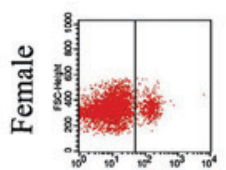

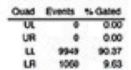

CD4 T Cells

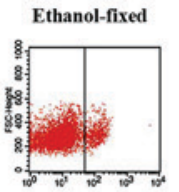

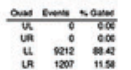

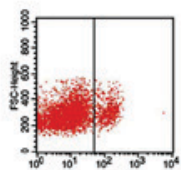

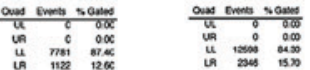

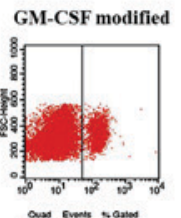

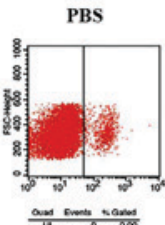
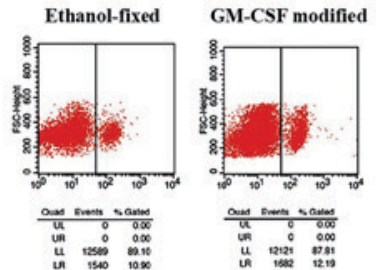

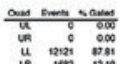

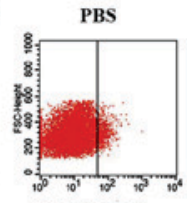

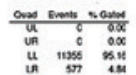

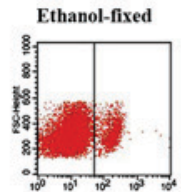

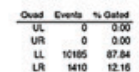
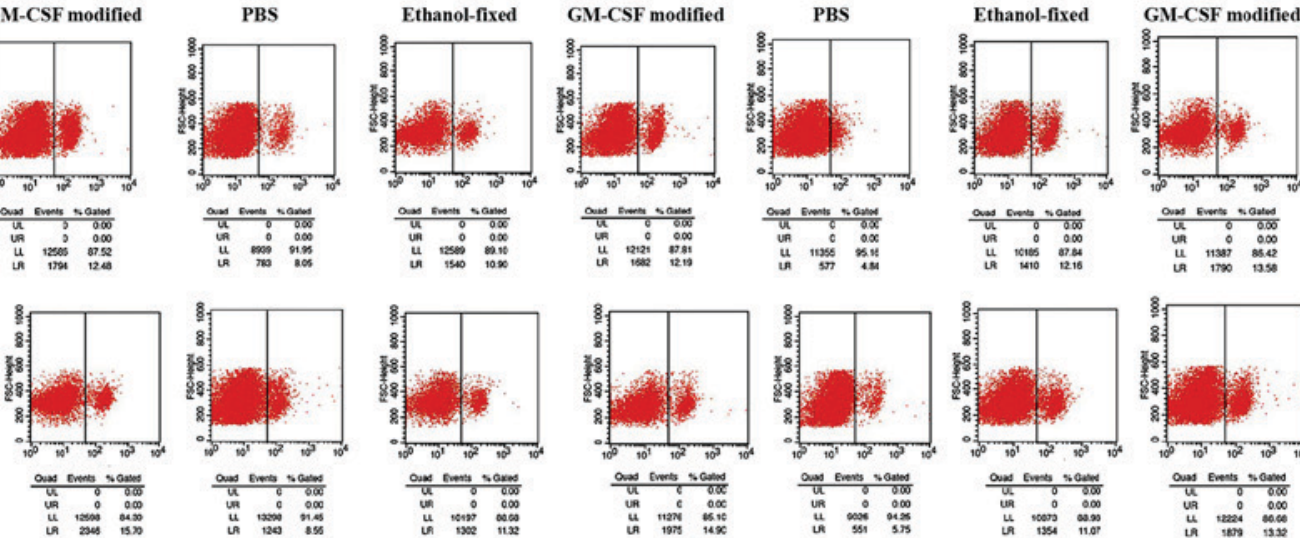

CD8 T Cells
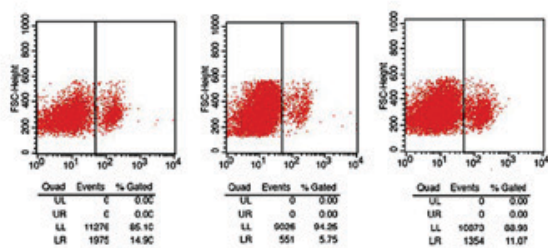

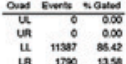

NK Cells

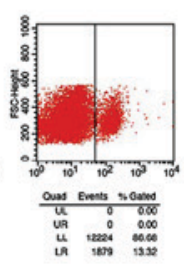

B
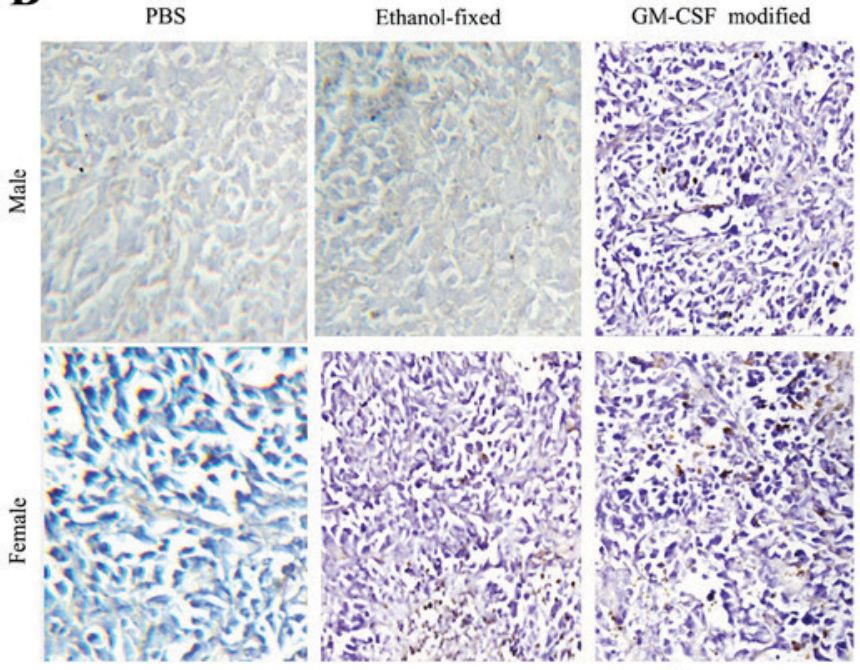

Infiltration of $\mathrm{CD}^{+} \mathrm{T}$ cells in tumor tissue
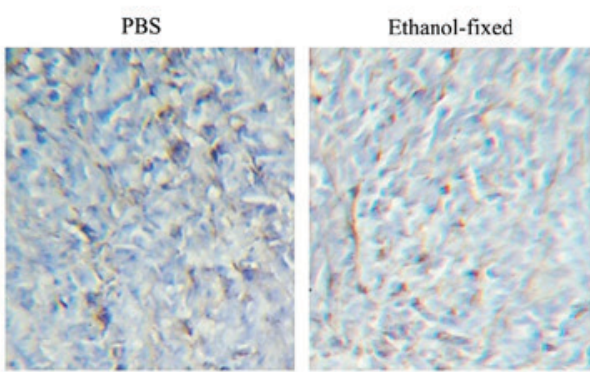

GM-CSF modified
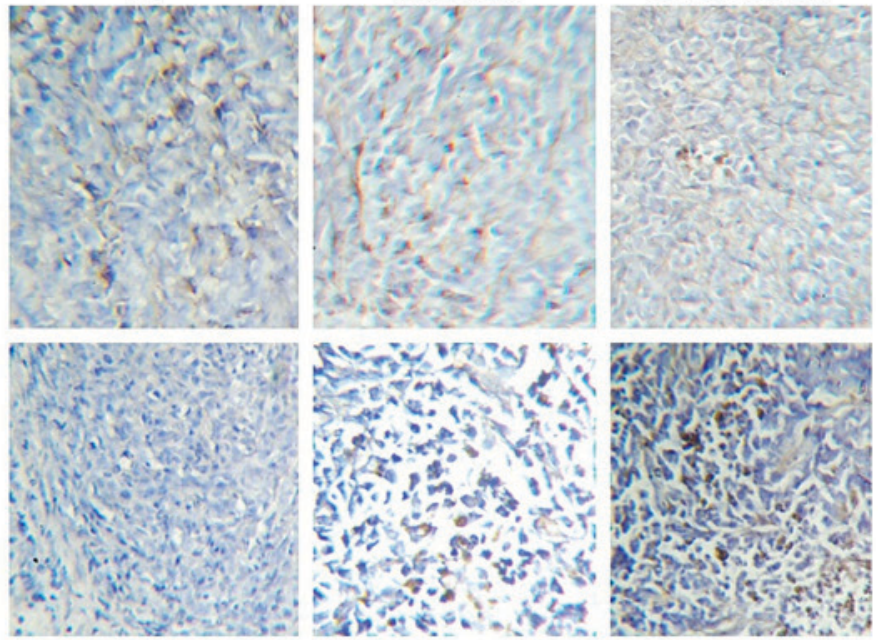

Infiltration of $\mathrm{CD}^{+} \mathrm{T}$ cells in tumor tissue

Figure 4. Analysis of lymphocytes in the spleens and tumors. (A) Splenoctytes were isolated 7 days after the final vaccination. Red blood cells were lysed with ammonia-chloride-potassium lysis buffer. Splenocytes were stained with FITC-labeled anti-mCD4, anti-mCD8 and anti-mCD161 antibodies for 1 h. CD4 ${ }^{+}$ $\mathrm{T}$ cells, $\mathrm{CD} 8^{+} \mathrm{T}$ cells, and NK cells were analyzed using flow cytometry. The number of $\mathrm{CD} 4^{+} \mathrm{T}$ cells and $\mathrm{CD} 8^{+} \mathrm{T}$ cells was highest in the spleens of female mice administered the GM-CSF-modified cell vaccine. By contrast, the male GM-CSF-modified cell vaccine groups exhibited the highest proportion of NK cells in the spleen. (B) Immunohistochemical investigation of how lymphocytes mediate the immune response. NK cells were undetectable in the tumor tissue from all groups. Infiltrating $\mathrm{CD} 4^{+} \mathrm{T}$ cells and $\mathrm{CD} 8^{+} \mathrm{T}$ cells were evaluated based on tumor histology, as described in the Materials and methods. The proportion of $\mathrm{CD}^{+} \mathrm{T}$ cells and $\mathrm{CD} 8^{+} \mathrm{T}$ cells within the tumors was highest in the female GM-CSF-modified cell vaccine groups. Magnification, $\mathrm{x} 200$. FITC, fluorescein isothiocyanate; CD4, cluster of differentiation 4; NK, natural killer; GM-CSF, granulocyte-macrophage colony-stimulating factor.

the immune system will become tolerant to the tumor cells. RM-1 prostate cancer cells express extremely low levels of MHCI and exhibit low immunogenicity, which makes it difficult for antigen-presenting cells to present tumor antigens to $\mathrm{CD} 8^{+} \mathrm{T}$ cells via the MHC I pathway, preventing $\mathrm{T}$ cells from inducing an immune response. In the female mouse model, such tumor tolerance does not exist, and the mice therefore generate a sustained and potent immune response against the cancer. This model may aid the acceleration of vaccine development for the clinic. The results of the present study revealed that in males, low numbers of NK cells mediated the antitumor immune response in the absence of CTLs. In vitro experiments using splenocytes from mice that received the GM-CSF-modified cell vaccine against RM-1 cells revealed that the secretion of IFN- $\gamma$ was lower in males compared with in females. Additionally, the proportion of $\mathrm{CD} 4{ }^{+} \mathrm{T}$ cells and
$\mathrm{CD} 8^{+} \mathrm{T}$ cells within the spleen and tumor tissue was lower in the male group that received the GM-CSF-modified cell vaccine group than in the equivalent female group. However, NK cells were undetectable in the tumor tissue from all experimental groups. All males were sacrificed within 35 days, and the survival curves did not differ significantly between each male treatment group. By contrast, in females, the data indicated that there was a 20\% tumor-free survival rate in the group that received the GM-CSF-modified RM-1 cell vaccine. The difference in the immune response was largely associated with sex differences. The prostate antigen was recognized as 'self' by the male immune system but as 'foreign' by the female immune system. The GM-CSF-modified RM-1 cell vaccine was seen as 'non-self' and was therefore recognized by the female immune system, which induced a strong response to clear the tumor. This model may be used to evaluate the 
effects of cell-based vaccines in females. In this model, female and male mice injected with viable RM-1 cells were used to identify differences in the immune response. An advantage of this model is that it may be used to study sex differences in the immune response, to identify approaches for improving protection from disease in males.

Owing to the lack of an effective model, it remains unclear how a whole-cell vaccine may induce an immune response against viable RM-1 cells. Several groups have reported that the antitumor immune response is mediated by NK cells, $\mathrm{CD}^{+} \mathrm{T}$ cells and $\mathrm{CD}^{+} \mathrm{T}$ cells $(22,23)$. Previous studies have reported that NK cells directly mediate the immune response, as $\mathrm{CD}^{+} \mathrm{T}$ cells are inhibited by $\mathrm{CD} 4^{+} \mathrm{CD} 25$-regulatory $\mathrm{T}$ cells $(13,19)$. Data from the present study revealed that in females, the antitumor immune response induced by the GM-CSF-modified cell vaccine was predominantly mediated by $\mathrm{CD}^{+} \mathrm{T}$ cells and, to a lesser extent, by NK cells. In males, a small number of NK cells were involved in the cytotoxic immune response in the early stage, while CTL lysis was undetectable. Additionally, in the two sexes, the supernatant from in vitro $\mathrm{CD}^{+} \mathrm{T}$ cell cultures exhibited RM-1-specific IFN- $\gamma$ production, but little IL-4 production. In short, clearance of the tumors required $\mathrm{CD} 8^{+} \mathrm{T}$ cells and $\mathrm{NK}$ cells, although $\mathrm{CD}^{+} \mathrm{T}$ cells predominantly mediated the antitumor response.

The present study demonstrated that between the sexes, there was a large difference in the immune response, as female mice were intolerant to prostate antigens. Thus, the model used in the present study is clinically relevant and may aid acceleration of the development of whole-cell vaccines. In addition, the model represents progress in the study of clinical immunotherapies for prostate cancer.

\section{References}

1. Siegel R, Ma J, Zou Z and Jemal A: Cancer statistics, 2014. CA Cancer J Clin 64: 9-29, 2014.

2. Crawford ED, Blumenstein BA, Goodman PJ, Davis MA, Eisenberger MA, McLeod DG, Spaulding JT, Benson R and Dorr FA: Leuprolide with and without flutamide in advanced prostate cancer. Cancer 66 (5 Suppl): S1039-S1044, 1990.

3. Quinn DI, Tangen CM, Hussain M, Lara PN Jr, Goldkorn A, Moinpour CM, Garzotto MG, Mack PC, Carducci MA, Monk JP, et al: Docetaxel and atrasentan versus docetaxel and placebo for men with advanced castration-resistant prostate cancer (SWOG S0421): A randomised phase 3 trial. Lancet Oncol 14: 893-900, 2013.

4. Armstrong AJ and Carducci MA: Chemotherapy for advanced prostate cancer: Results of new clinical trials and future studies. Curr Oncol Rep 7: 220-227, 2005.

5. Haigh PI, Difronzo LA, Gammon G and Morton DL: Vaccine therapy for patients with melanoma. Oncology (Williston Park) 13: 1561-1574, 1999.

6. Li H, Jiang HJ, Ma MQ, Wei F, An XM and Ren XB: Vaccination with allogeneic GM-CSF gene-modified lung cancer cells: Antitumor activity comparing with that induced by autologous vaccine. Cancer Biother Radiopharm 22: 790-798, 2007.
7. Obata C, Zhang M, Moroi Y, Hisaeda H, Tanaka K, Murata S, Furue M and Himeno K: Formalin-fixed tumor cells effectively induce antitumor immunity both in prophylactic and therapeutic conditions. J Dermatol Sci 34: 209-219, 2004

8. Yin W, He Q, Hu Z, Chen Z, Qifeng M, Zhichun S, Zhihui Q, Xiaoxia N, Li J and Gao J: A novel therapeutic vaccine of GM-CSF/TNFalpha surface-modified RM-1 cells against the orthotopic prostatic cancer. Vaccine 28: 4937-4944, 2010.

9. Suckow MA, Wolter WR and Pollard M: Prevention of de novo prostate cancer by immunization with tumor-derived vaccines. Cancer Immunol Immunother 54: 571-576, 2005.

10. Kotera Y,Shimizu K and Mulé JJ: Comparative analysis of necrotic and apoptotic tumor cells as a source of antigen(s) in dendritic cell-based immunization. Cancer Res 61: 8105-8109, 2001.

11. Ahram M, Flaig MJ, Gillespie JW, Duray PH, Linehan WM, OrnsteinDK,NiuS,ZhaoY,PetricoinEFIIIandEmmert-BuckMR: Evaluation of ethanol-fixed, paraffin-embedded tissues for proteomic applications. Proteomics 3: 413-421, 2003.

12. Baley PA, Yoshida K, Qian W, Sehgal I and Thompson TC: Progression to androgen insensitivity in a novel in vitro mouse model for prostate cancer. J Steroid Biochem Mol Biol 52: 403-413, 1995.

13. Griffith TS, Kawakita M, Tian J, Ritchey J, Tartaglia J, Sehgal I, Thompson TC, Zhao W and Ratliff TL: Inhibition of murine prostate tumor growth and activation of immunoregulatory cells with recombinant canarypox viruses. J Natl Cancer Inst 93: 998-1007, 2001.

14. Labarthe MC, Theocharous P, Russell N, Todryk S, Bangma C Thraves P, Dalgleish AG and Whelan MA: A novel murine model of allogeneic vaccination against prostate cancer. Cancer Immunol Immunother 57: 453-465, 2008.

15. He Q, Li J, Yin W, Song Z, Zhang Z, Yi T, Tang J, Wu D, Lu Y, Wang Z, et al: Low-dose paclitaxel enhances the anti-tumor efficacy of GM-CSF surface-modified whole-tumor-cell vaccine in mouse model of prostate cancer. Cancer Immunol Immunother 60: 715-730, 2011.

16. Cooper MD and Alder MN: The evolution of adaptive immune systems. Cell 124: 815-822, 2006.

17. Li M, Davey GM, Sutherland RM, Kurts C, Lew AM, Hirst C, Carbone FR and Heath WR: Cell-associated ovalbumin is cross-presented much more efficiently than soluble ovalbumin in vivo. J Immunol 166: 6099-6103, 2001.

18. Gallucci S, Lolkema $M$ and Matzinger P: Natural adjuvants: Endogenous activators of dendritic cells. Nat Med 5: 1249-1255, 1999.

19. Sauter B, Albert ML, Francisco L, Larsson M, Somersan S and Bhardwaj N: Consequences of cell death: Exposure to necrotic tumor cells, but not primary tissue cells or apoptotic cells, induces the maturation of immunostimulatory dendritic cells. J Exp Med 191: 423-434, 2000.

20. GrantJF, Iwasawa T,SinnHW,Siemens DR, Griffith TS, Takacs EB and Ratliff TL: Induction of protective immunity to RM-1 prostate cancer cells with ALVAC-IL-2/IL-12/TNF-alpha combination therapy. Int J Cancer 119: 2632-2641, 2006.

21. Carbone FR, Kurts C, Bennett SR, Miller JF and Heath WR: Cross-presentation: A general mechanism for CTL immunity and tolerance. Immunol Today 19: 368-373, 1998.

22. Chhikara M, Huang H, Vlachaki MT, Zhu X, Teh B, Chiu KJ, Woo S, Berner B, Smith EO, Oberg KC, et al: Enhanced therapeutic effect of HSV-tk+GCV gene therapy and ionizing radiation for prostate cancer. Mol Ther 3: 536-542, 2001.

23. Schroten-Loef C, de Ridder CM, Reneman S, Crezee M, Dalgleish A, Todryk SM, Bangma CH and Kraaij R: A prostate cancer vaccine comprising whole cells secreting IL-7, effective against subcutaneous challenge, requires local GM-CSF for intra-prostatic efficacy. Cancer Immunol Immunother 58: 373-381, 2009. 